\title{
Changes in the age-specific body mass index distribution among urban children between 2002 and 2018 in Changsha, China
}

\author{
Sha Zhao ${ }^{1 \#}$, Xun Li ${ }^{2 \#}$, Shi-Ting Xiang ${ }^{2}$, Lihua Xie ${ }^{3}$, Rutong Kang ${ }^{2}$, Liping Li $^{2}$, Zhenghui Xiao ${ }^{4}$, \\ Yan Zhong $^{1 \wedge}$ \\ ${ }^{1}$ Institute of Child Health, Hunan Children's Hospital, Changsha, China; ${ }^{2}$ Pediatrics Research Institute of Hunan Province, Hunan Children's \\ Hospital, Changsha, China; ${ }^{3}$ Outpatient Department, Hunan Children's Hospital, Changsha, China; ${ }^{4}$ Hunan Province Key Lab of Pediatric \\ Emergency Medicine, Hunan Children's Hospital, Changsha, China \\ Contributions: (I) Conception and design: S Zhao, X Li, Y Zhong; (II) Administrative support: S Zhao, L Xie, R Kang, L Li, Z Xiao, Y Zhong; (III) \\ Provision of study materials or patients: S Zhao, L Xie, R Kang, L Li, Z Xiao, Y Zhong; (IV) Collection and assembly of data: S Zhao, X Li, SX, L \\ Xie, R Kang, L Li, Z Xiao, Y Zhong; (V) Data analysis and interpretation: All authors; (VI) Manuscript writing: All authors; (VII) Final approval of \\ manuscript: All authors. \\ "These authors contributed equally to this work. \\ Correspondence to: Yan Zhong. Institute of Child Health, Hunan Children's Hospital, 86 Ziyuan Road, Changsha 410007, China. \\ Email: yz_hnch@163.com.
}

Background: It had been reported that the increase in the prevalence of childhood overweight and obesity in several developed countries over the decade was largely a consequence of changes in the upper end of the body mass index (BMI) distribution. We speculated that the change in BMI distribution among Chinese children is different from that in developed countries. Therefore, we investigated the changes in the BMI distribution among urban children in Changsha, China.

Methods: A secondary data analysis of the 2002 and 2018 Annual Survey on Students' Constitution and Health from Yuhua District, Changsha City, China was performed. The BMI distribution of children aged 6-15 years $(n=72,061)$ was examined by calculating the BMI value at 5 th, 50th, 85 th, and 95 th percentiles. Differences in the BMI value at the 5th, 50th, 85th, and 95th percentiles between 2002 and 2018 according to age and sex were calculated.

Results: From 2002 to 2018, the standardized rate of underweight decreased from $6.9 \%$ to $3.4 \%$, whereas that of obesity increased from $6.1 \%$ to $13.8 \%$. The BMI of the population increased over the 16 -year period. The greatest change between the years was seen in the 85 th percentile, and moderate changes were seen in the 5th and 50th BMI percentiles. Also, the greatest changes in the BMI percentiles were evident at age $12-14$ years.

Conclusions: The whole spectrum of BMI distribution among school-aged children shifted over the 16-year study period, although the increases were more marked in the upper than lower percentiles. Health professionals and health policymakers should develop strategies and interventions aimed at preventing adverse health effects caused by this change.

Keywords: Children; body mass index (BMI); distribution; obesity; overweight

Submitted Sep 22, 2020. Accepted for publication Jan 17, 2021.

doi: $10.21037 /$ tp-20-305

View this article at: http://dx.doi.org/10.21037/tp-20-305

^ ORCID: Xun Li, 0000-0002-5070-8950; Zhenghui Xiao, 0000-0003-2056-8798; Yan Zhong, 0000-0003-1132-5567. 


\section{Introduction}

Together with socioeconomic development and transformation of lifestyle, the childhood obesity epidemic in different part of this world has changed over time (1-4). Although the childhood obesity rate has plateaued in some developed countries, it is increasing in rapidly developing countries, such as China $(5,6)$. The prevalence of overweight and obesity among school-aged children in China has increased from $5.3 \%$ in 1995 to $20.5 \%$ in 2014 (7).

Understanding how the BMI distribution changes over time can allow identification of subpopulations susceptible to overweight and obesity, enabling the development of tailored interventions $(8,9)$. Studies had investigated the changes in the shape and location of BMI distributions over the last decades in several developed countries, and found the most pronounced elevations in BMI have occurred in the upper part of the BMI spectrum ( $>75$ th percentiles), whereas little or no changes have occurred below the 50th percentile (10-14). These studies concluded that the increases in the mean BMI and prevalence of overweight and obesity in children have resulted mainly from the pronounced change in the upper end of the BMI spectrum, suggesting that the factors leading to BMI changes were changed in only a subgroup of the schoolaged population (10-12).

However, we speculated that the pattern of change in the BMI distribution in China is different from that in developed countries, because the increased obesity prevalence in China is accompanied by reduced rates of stunting and thinness $(7,15)$; therefore, the entire spectrum of BMI distribution may have shifted. The aim of this study was to investigate the changes in the BMI distribution of Chinese children overtime and identify the age at which the BMI distribution is most sensitive to change.

We chose the Yuhua District of Changsha City as the study site. Changsha is a second-tier city located in central south China. The lifestyle in Changsha has changed dramatically over the past two decades as a result of rapid socioeconomic development. Yuhua District is one of six districts of Changsha City, and it covers 292 square kilometers. The gross domestic product of Yuhua District increased from $¥ 2,425$ million in 2002 to $¥ 188,816$ million in 2018. We investigated the prevalence of underweight, overweight, and obesity among children aged 6-15 years in Yuhua District in 2002 and 2018. The distribution of BMI was examined as the BMI values at 5 th, 50 th, 85 th, and 95 th percentiles. And the changes of BMI percentiles over time were calculated according to age and sex.
We present the following article in accordance with the STROBE reporting checklist (available at http://dx.doi. org/10.21037/tp-20-305).

\section{Methods}

\section{Study setting and participants}

This study was a secondary data analysis of the 2002 and 2018 Annual Survey on Students' Constitution and Health from Yuhua District, Changsha City, China. The survey was sponsored by the Education Bureau of Changsha City. The participants were primary or middle school students. A random cluster sampling method was used to select schools from Yuhua District. All students at the selected schools underwent standard physical examinations. Children aged less than 6 years or more than 15 years from the dataset were excluded from the analysis. In China, school-aged children must complete nine years of compulsory education in primary (6 years) and middle (3 years) school, starting primary school at 6 to 7 years of age. Therefore, surveys in primary and middle schools take into account the majority (>98\%) of children 6-15 years of age in the study region.

The authors are accountable for all aspects of the work in ensuring that questions related to the accuracy or integrity of any part of the work are appropriately investigated and resolved. The study protocol was reviewed and approved by the Medical Ethics Committee of the Hunan Children's Hospital (HCHLL-2019-41). The study was conducted in accordance with the Declaration of Helsinki (as revised in 2013). Informed consent was waived because this was a retrospective study, and the authors had no access to information that could identify individual participants during and after data collection.

\section{Measurements and variables}

Age (years), sex, height, and weight were collected from the Annual Survey on Students' Constitution and Health dataset. Height and weight were measured by a team of health professionals, following the Handbook of National Student Constitution and Health Survey. BMI was calculated as weight in kilograms divided by the square of height in meters. The weight status of children was defined according to BMI percentile based on growth charts for Chinese children and adolescents aged 0-18 years (16). The growth charts provide age and sex-specific BMI cutoff values for underweight (BMI $<5$ th percentile), healthy 
Table 1 Sample size of the study population according to sex and survey year, Yuhua District, Changsha, China

\begin{tabular}{ccc}
\hline \multirow{2}{*}{ Sex and age (years) } & 2002 & 2018 \\
\cline { 2 - 3 } All participants & & \\
$6-15$ & 26,644 & 45,417 \\
$6-10$ & 14,345 & 25,310 \\
$11-15$ & 12,299 & 20,107 \\
Males & & \\
$6-15$ & 14,127 & 24,214 \\
$6-10$ & 7,570 & 13,289 \\
$11-15$ & 6,557 & 10,925 \\
Females & & \\
$6-15$ & 12,517 & 21,203 \\
$6-10$ & 6,775 & 12,021 \\
$11-15$ & 5,742 & 9,182 \\
\hline
\end{tabular}

weight (BMI 5th-<85th percentile), overweight (BMI 85th$<95$ th percentile), and obese (BMI $\geq 95$ th percentile) $(16,17)$.

\section{Statistical analysis}

Crude rates and $95 \%$ confidence intervals (CIs) for each weight status were calculated by sex and survey year. Standardized rates for each weight status were estimated using the sum of the 2002 and 2018 populations as the standard population and were adjusted by age and sex. All hypothesis tests were two tailed with the type 1 error rate fixed at $5 \%$. Percentiles (5th, 50th, 85 th, and 95 th) were used to examine the distribution of BMI. The differences on the BMI value at 5 th, 50th, 85 th, and 95 th percentiles between 2002 and 2018 were calculated according to age and sex, and the difference in percent were also calculated. Missing data were not imputed. Statistical analysis was conducted using SAS version 9.3 software (SAS Institute, Cary, NC, USA).

\section{Sensitivity analysis}

Because yearly data from 2002 to 2018 were not available, we used data from two years (2002 and 2018). To assess the effect of the study year (selection bias) on the results, sensitivity analyses were conducted using data from the
2003 and 2017 Annual Survey on Students' Constitution and Health. As data for children aged 6 and 15 years were not available in the 2003 dataset, the sensitivity analyses were performed in children aged 7-14 years. The standardized rates of the weight status; the age and sexspecific prevalence of underweight, overweight and obesity; and the BMI at 5th, 50th, 85th, and 95 th percentile by age and sex in 2003 and 2017 were calculated.

\section{Results}

The sample sizes of the two surveys are shown in Table 1 . In 2002 and 2018, 26,644 and 45,417 children 6-15 years of age, respectively, participated in the health survey. All participants had complete records for age, sex, height, and weight; therefore, no participants were excluded because of missing data.

The distributions of weight status according to sex and survey year are presented in Table 2 . The standardized rate of underweight decreased from $6.9 \%$ in 2002 to $3.4 \%$ in 2018 , and that of overweight increased from $7.3 \%$ in 2002 to $12.4 \%$ in 2018 . The prevalence of obesity increased from $6.1 \%$ in 2002 to $13.8 \%$ in 2018 .

Figure 1 shows the prevalence of underweight, overweight, and obesity according to age, sex, and survey year. In 2002, the prevalence of underweight increased markedly after age 10 years in both sexes, peaked at age 13 years, and decreased thereafter. The prevalence of underweight in 2018 reached a plateau at aged 6-11 years, and then decreased to $<3.5 \%$ in both sexes.

The prevalence of overweight and obesity (BMI $\geq 85$ th) were generally lower in 2002 than in 2018 (Figure 1). The prevalence of overweight and obesity decreased from ages 6 to 15 years in 2002, but increased from ages 6 to 10 years and decreased with age thereafter in 2018. The obesity prevalence followed a similar pattern.

Figure 2 shows the BMI values at 5 th, 50 th, 85 th, and 95th percentile according to age, sex, and survey year. The BMI distributions were more skewed to the right in 2018 than in 2002. From 2002 to 2018, increases in the values of BMI at all percentiles were observed for both sexes. The differences between two survey years were greater at 85 th and 95 th than at the 5 th and 50th percentiles. Marked differences in BMI value occurred at a younger age in the 50th percentile compared with the 5 th percentile.

Table 3 shows the differences in values, as well as differences in percent at the 5 th, 50th, 85 th, and 95 th BMI percentile between 2002 and 2018 according to sex. The 5th 
Table 2 Distribution of weight status according to sex and survey year among children 6-15 years

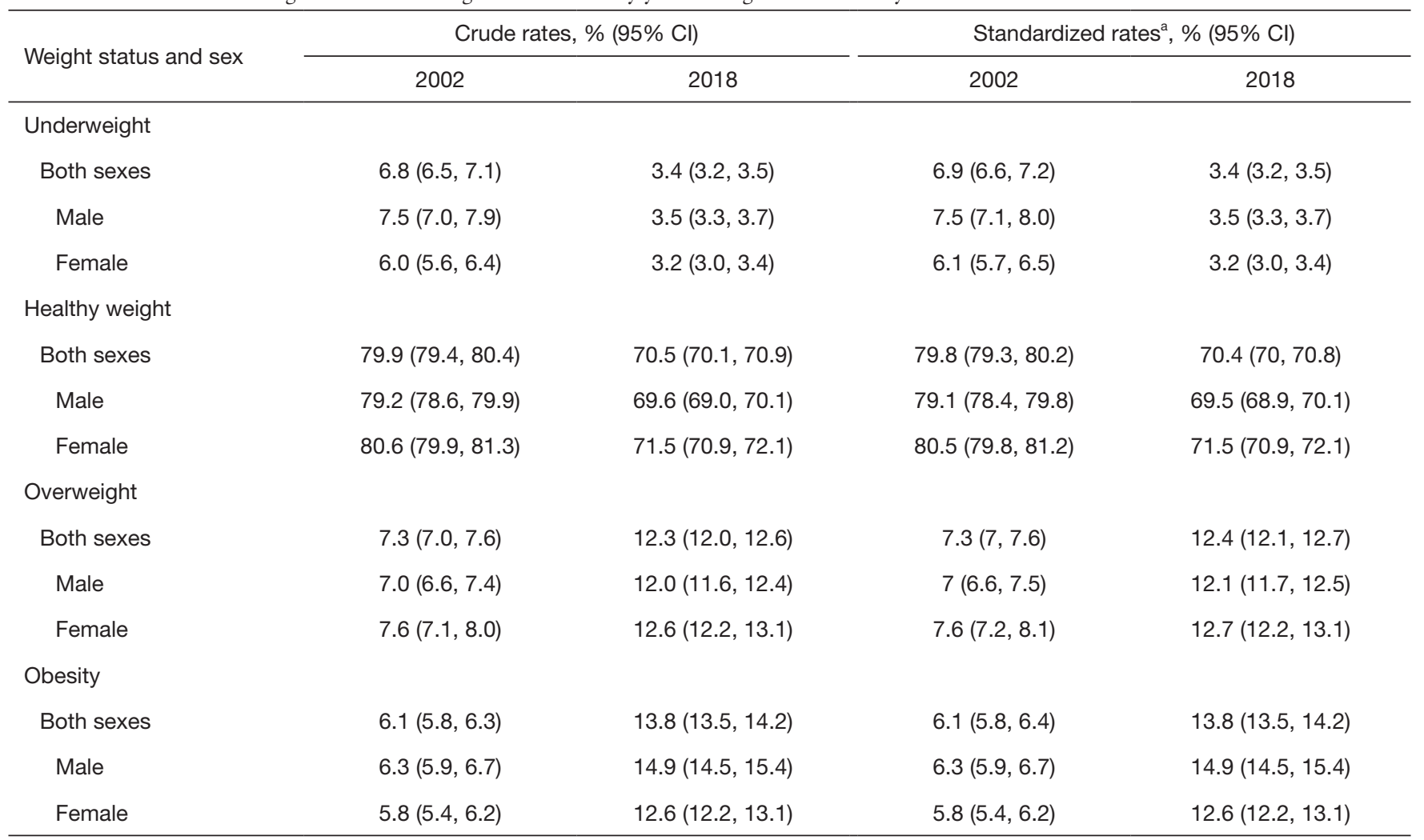

${ }^{a}$, rates were adjusted by age and sex, using the sum of both population as the standard population. Cl, confidence interval.
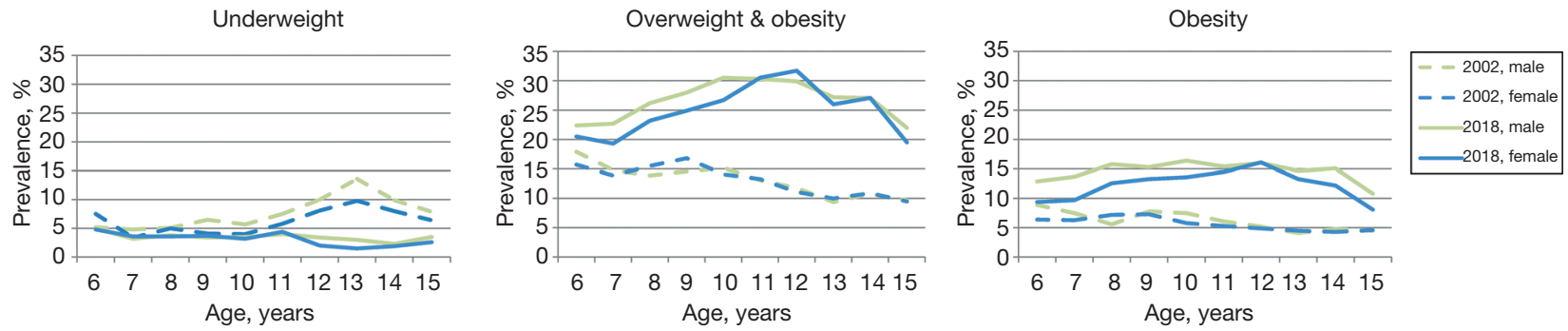

Figure 1 Age and sex-specific prevalence of underweight, overweight, and obesity among children in 2002 and 2018 , Yuhua District, Changsha, China.

BMI percentile was moderately elevated from 2002 to 2018 in most ages, with most growths less than $10 \%$. In males, the BMI at 50th percentile increased by $>10 \%$ after 11 years of age. For female, the growth of BMI at 50th percentile increased until the age of 12 years $(+12.27 \%)$, and slowed down thereafter. The greatest change in BMI from 2002 to 2018 was in the 85 th percentile, with differences in percent ranging from $2.8 \%$ to $19.7 \%$ among male, and from $2.2 \%$ to $14.8 \%$ among female. Substantial increases in BMI were also observed at the 95th percentiles, with growth in percent ranging from $4.2 \%$ to $15.2 \%$ among male, and from $3.6 \%$ to $14.2 \%$ among female. Overall, the greatest change in the value of BMI percentiles occurred at age 12-14 years.

\section{Sensitivity analysis}

Sensitivity analyses were conducted using data from the 2003 and 2017 Annual Survey on Students' Constitution 

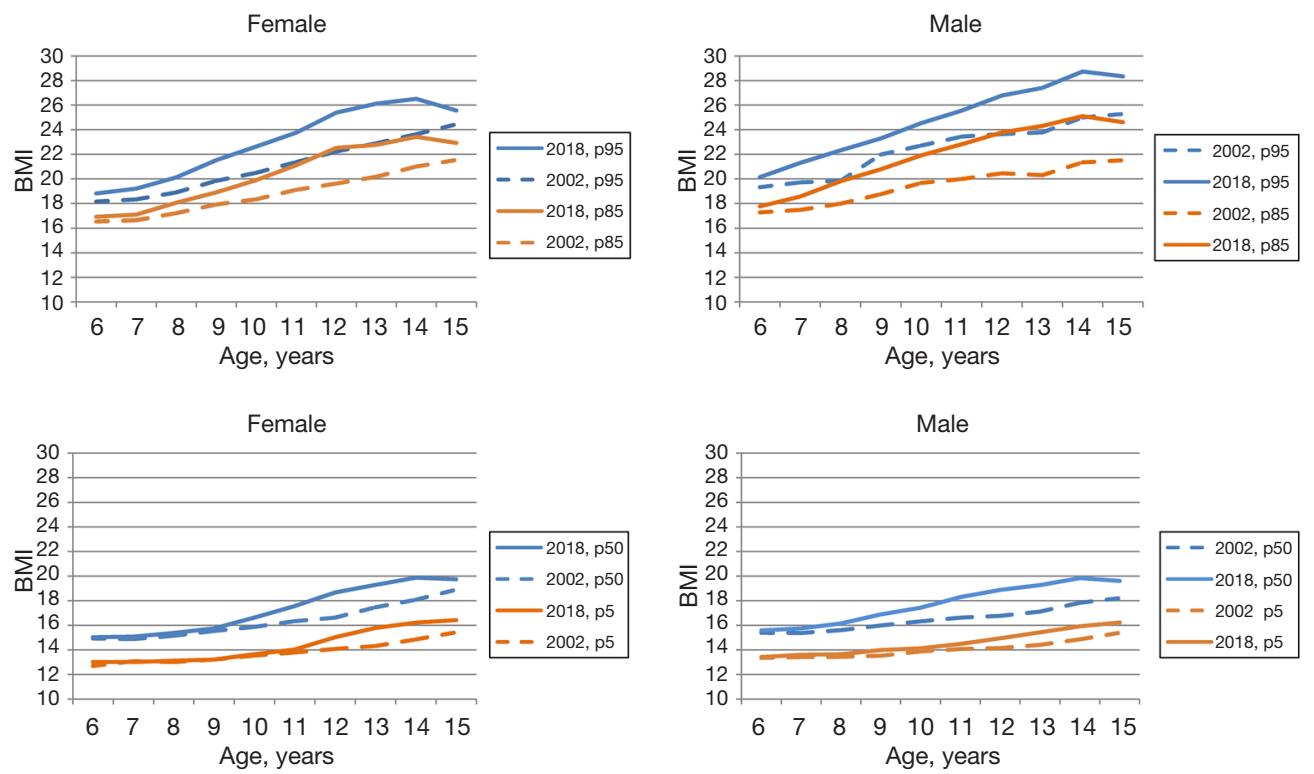

Figure 2 Distribution of BMI at 5th, 50th, 85th, and 95th percentile according to age and sex among children in 2002 and 2018, Yuhua District, Changsha, China. p5, 5th percentile; p50, 50th percentile; p85, 85th percentile; p95, 95th percentile. BMI, body mass index.

and Health. The standardized rate of underweight decreased from $6.2 \%$ in 2003 to $3.8 \%$ in 2017 , while the prevalence of overweight increased from $8.6 \%$ in 2003 to $11.7 \%$ in 2017 , and the prevalence of obesity increased from $8.1 \%$ in 2003 to $13.1 \%$ in 2017 (Table S1). The distributions of the age and sex-specific prevalence of underweight, overweight and obesity in 2003 and 2017 are presented in Figure S1. The BMI at 5th, 50th, 85th, and 95th percentile according to age and sex in 2003 and 2017 are shown in Figure S2. The differences in the age and sex-specific prevalence of three weight statuses and in the BMI percentiles between 2003 and 2017 were similar to those differences between 2002 and 2018, but in a relatively more gradual and gentle manner.

\section{Discussion}

The prevalence of underweight decreased from $6.8 \%$ in 2002 to $3.4 \%$ in 2018 among children $11-15$ years of age, and that obesity increased from $6.1 \%$ to $13.8 \%$. The BMI of the entire population increased over the 16-year study period in both sexes, and the greatest change was seen in the 85 th percentile, with moderate changes seen in the 5th and 50th percentiles. As for the age specific changes of BMI distribution, the greatest changes occurred at the 12-14 years of age.

Our findings of increased prevalence of overweight and obesity, as well as the decreased prevalence of underweight over the 16-year period are consistent with other studies in Chinese populations (15,18-21). Because of regional disparities in socioeconomic development in China, the epidemic of overweight and obesity started to emerge in all parts of China with different speed (20). Without immediate interventions, it could be foreseen that the increasing trends in the prevalence of overweight and obesity in China would be sustained in the years to come.

Comparing the age specific BMI distributions over time can pinpoint the ages at which $\mathrm{BMI}$ is sensitive to change, allowing identification of the susceptible age groups to launch interventions. In this study, the changes of BMI in percentiles increased with age in both sexes. Similar patterns had been observed in the 95th percentile among children in the United States, 1988-1994 through 2013-2014 (13). Whether this is because the BMI of older children are more sensitive to lifestyle changes or a result of a cumulative effect of lifestyle changes on weight status is unclear. However, our data suggest the need for interventions to prior to 12 years of age.

Studies in several developed countries showed that the increases in mean BMI and prevalence of obesity were caused by an increase of BMI among children who were in the upper BMI percentiles (10-14). For example, Kurokawa et al. analyzed the trends of BMI distribution in Japanese 
Table 3 Changes in BMI percentiles from 2002 to 2018 according to sex and age, Yuhua District, Changsha, China

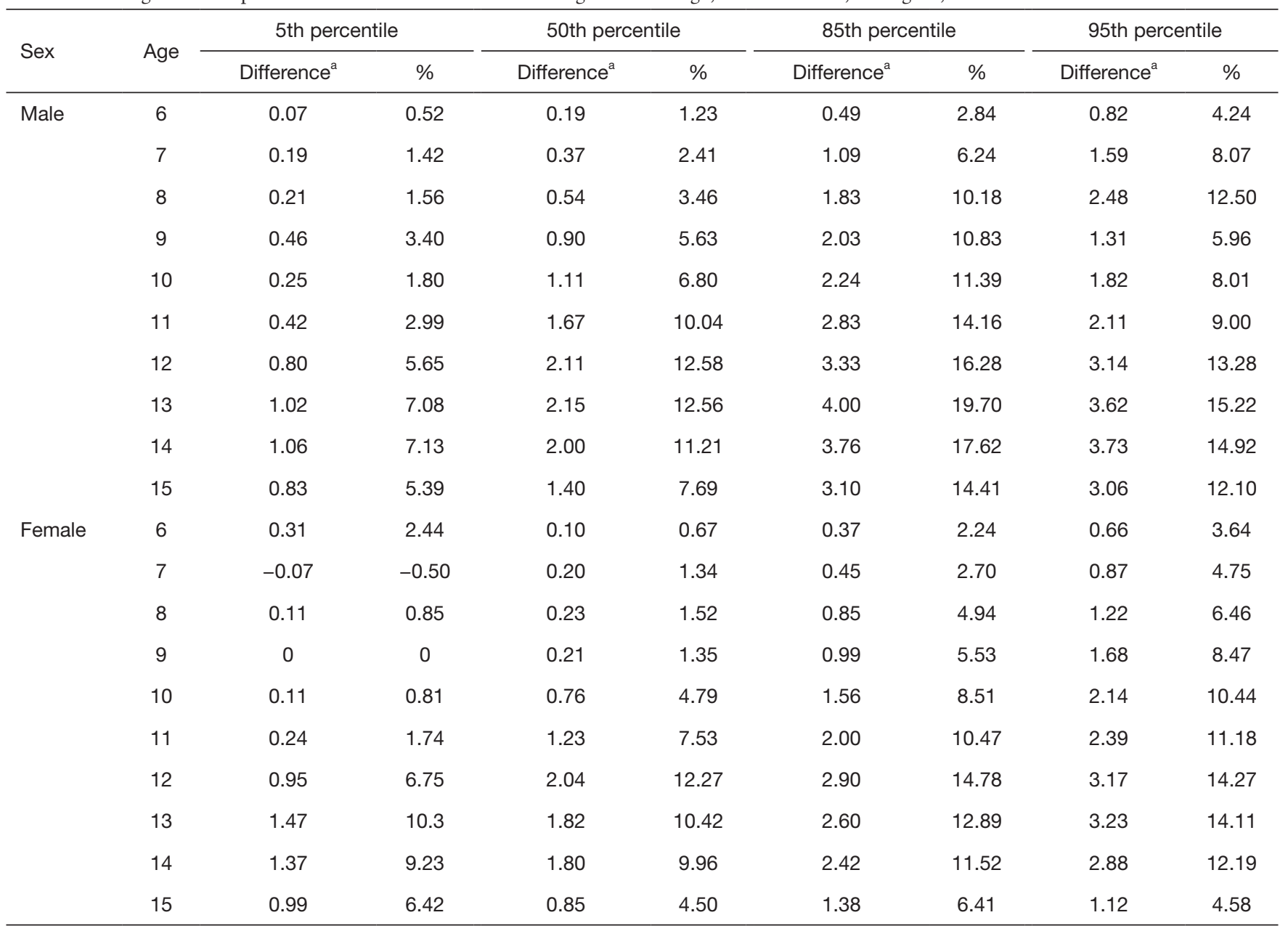

a, unit: $\mathrm{kg} / \mathrm{m}^{2}$. BMl, body mass index.

children from 1989 to 2003, and found little or no change in the 10th and 25th percentiles. However, the increases in the 75th and 90th percentiles were more marked than that in the 50th percentile among primary school children (11). The authors speculated that a subgroup of the population (in the upper percentiles of $\mathrm{BMI})$ is more susceptible to factors that increase the risk of overweight and obesity (11). However, we found a shift in the BMI distribution in the entire population, although the increases in the upper percentiles were more marked than those in the lower percentiles. Unlike in developed countries, the BMI shift in this study indicated that the factors that contributing to the increased BMI influenced the entire population. This finding emphasizes the need for studies on the short- and long-term effect of increasing BMI on population health.

The rapid socioeconomic development in China over the past two decades has led to a dramatic change in lifestyle, resulting in an obesity epidemic $(22,23)$. This change is now affecting the health of the younger generation $(7,22)$. The increase in BMI among children of all ages and in all BMI percentiles was unsurprising and indicates the need for actions to prevent adverse consequences. The health consequence of the shift in BMI distribution should be investigated, and interventions are needed to slow the increasing obesity prevalence and prevent related adverse health effects.

This study had several limitations. First, BMI was used to evaluate overweigh and obesity. Although BMI is an imperfect measure of body fat and adiposity $(24,25)$, it is the most feasible and widely used measurement in public health surveillance $(1,7,17,26,27)$. The weight status of children and teenagers is defined according to BMI percentiles for 
age and sex. As shown in this study, the BMI distribution is changing, meaning that BMI percentile values are not fixed. To make the distribution of weight status comparable between 2002 and 2018, fixed cutoff points to define weight status are needed (26). Therefore, we used the cutoffs from the growth charts delivered in 2009 (16), which are still in use in China in 2020. Second, as a retrospective study, we analyzed available data only and thus could not evaluate factors associated with the BMI change. Also, as a crosssectional study, we could not analyze the BMI trajectory. Predicting age-specific trends in BMI shifting and developing targeted interventions will require identification of the associated factors and their effect sizes. Therefore, a further longitudinal study is needed. Third, this study used cross-sectional data from 2002 and 2018, and not the yearly data in between; therefore, we could not investigate the pattern and changing trend in BMI over the 16-year period. To predict future changes in BMI and evaluate the effectiveness of interventions, annual changes in BMI distribution need to be monitored. Fourth, this study was conducted in a single district in China, limiting the generalization of the findings. Further studies are needed to investigate whether other regions in China and other developing countries are undergoing similar transitions in BMI distribution, and what kind of interventions are needed to prevent adverse health consequences.

\section{Conclusions}

The whole spectrum of BMI distribution among schoolaged children in Yuhua District was shifted over the 16year study period in both sexes. The increases in BMI were moderate at the 5 th and 50th percentiles, and was greatest at 85 th percentile. Further studies should investigate the short- and long-term effects of increasing BMI on population health, and interventions to prevent the adverse health effects of increased BMI are needed.

\section{Acknowledgments}

Funding: This work was supported by the Science Foundation of Hunan Children's Hospital (2019-A).

\section{Footnote}

Reporting Checklist: The authors have completed the STROBE reporting checklist. Available at http://dx.doi. org/10.21037/tp-20-305
Data Sharing Statement: Available at http://dx.doi. org/10.21037/tp-20-305

Conflicts of Interest: All authors have completed the ICMJE uniform disclosure form (available at http://dx.doi. org/10.21037/tp-20-305). The authors have no conflicts of interest to declare.

Etbical Statement: The authors are accountable for all aspects of the work in ensuring that questions related to the accuracy or integrity of any part of the work are appropriately investigated and resolved. The study protocol was reviewed and approved by the Medical Ethics Committee of the Hunan Children's Hospital (HCHLL-2019-41). The study was conducted in accordance with the Declaration of Helsinki (as revised in 2013). The requirement for written informed consent was waived by the Medical Ethics Committee of the Hunan Children's Hospital.

Open Access Statement: This is an Open Access article distributed in accordance with the Creative Commons Attribution-NonCommercial-NoDerivs 4.0 International License (CC BY-NC-ND 4.0), which permits the noncommercial replication and distribution of the article with the strict proviso that no changes or edits are made and the original work is properly cited (including links to both the formal publication through the relevant DOI and the license). See: https://creativecommons.org/licenses/by-nc-nd/4.0/.

\section{References}

1. Ng M, Fleming T, Robinson M, et al. Global, regional, and national prevalence of overweight and obesity in children and adults during 1980-2013: a systematic analysis for the Global Burden of Disease Study 2013. Lancet 2014;384:766-81.

2. de Onis M, Blossner M, Borghi E. Global prevalence and trends of overweight and obesity among preschool children. Am J Clin Nutr 2010;92:1257-64.

3. Wang $\mathrm{Y}, \mathrm{Lim} \mathrm{H}$. The global childhood obesity epidemic and the association between socio-economic status and childhood obesity. Int Rev Psychiatry 2012;24:176-88.

4. NCD Risk Factor Collaboration. Worldwide trends in body-mass index, underweight, overweight, and obesity from 1975 to 2016: a pooled analysis of 2416 populationbased measurement studies in 128.9 million children, adolescents, and adults. Lancet 2017;390:2627-42. 
5. Wabitsch M, Moss A, Kromeyer-Hauschild K. Unexpected plateauing of childhood obesity rates in developed countries. BMC Med 2014;12:17.

6. Olds T, Maher C, Zumin S, et al. Evidence that the prevalence of childhood overweight is plateauing: data from nine countries. Int J Pediatr Obes 2011;6:342-60.

7. Dong Y, Jan C, Ma Y, et al. Economic development and the nutritional status of Chinese school-aged children and adolescents from 1995 to 2014: an analysis of five successive national surveys. Lancet Diabetes Endocrinol 2019;7:288-99. Erratum in: Lancet Diabetes Endocrinol. 2019 May;7(5):e5. doi: 10.1016/S2213-8587(19)30110-X. Epub 2019 Mar 20.

8. Hayes A, Gearon E, Backholer K, et al. Age-specific changes in BMI and BMI distribution among Australian adults using cross-sectional surveys from 1980 to 2008. Int J Obes (Lond) 2015;39:1209-16.

9. Benjamin Neelon SE, Ostbye T, Hales D, et al. Preventing childhood obesity in early care and education settings: lessons from two intervention studies. Child Care Health Dev 2016;42:351-8.

10. Eriksson M, Rasmussen F, Nordqvist T. Changes in shape and location of BMI distributions of Swedish children. Acta Paediatr 2005;94:1558-65.

11. Kurokawa N, Nakai K, Suzuki K, et al. Trends of body mass index distribution in schoolchildren in Sendai, Japan, 1989-2003. Obes Res Clin Pract 2009;3:1-52.

12. Ekblom O, Oddsson K, Ekblom B. Prevalence and regional differences in overweight in 2001 and trends in BMI distribution in Swedish children from 1987 to 2001. Scand J Public Health 2004;32:257-63.

13. Ogden CL, Carroll MD, Lawman HG, et al. Trends in Obesity Prevalence Among Children and Adolescents in the United States, 1988-1994 Through 2013-2014. JAMA 2016;315:2292.

14. Kalies H, Lenz J, von Kries R. Prevalence of overweight and obesity and trends in body mass index in German pre-school children, 1982-1997. Int J Obes Relat Metab Disord 2002;26:1211-7.

15. Song Y, Agardh A, Ma J, et al. National trends in stunting, thinness and overweight among Chinese school-aged children, 1985-2014. Int J Obes (Lond) 2019;43:402-11.

16. Li H, Ji CY, Zong XN, et al. Body mass index growth curves for Chinese children and adolescents aged 0 to 18 years. Zhonghua Er Ke Za Zhi 2009;47:493-8.

17. Ogden CL, Flegal KM. Changes in terminology for childhood overweight and obesity. Natl Health Stat Report 2010;(25):1-5.

18. Zhai L, Dong Y, Bai Y, et al. Trends in obesity, overweight, and malnutrition among children and adolescents in Shenyang, China in 2010 and 2014: a multiple crosssectional study. BMC Public Health 2017;17:151.

19. Zhang YX, Wang ZX, Zhao JS, et al. Trends in overweight and obesity among rural children and adolescents from 1985 to 2014 in Shandong, China. Eur J Prev Cardiol 2016;23:1314-20.

20. Jia P, Xue H, Zhang J, et al. Time Trend and Demographic and Geographic Disparities in Childhood Obesity Prevalence in China-Evidence from Twenty Years of Longitudinal Data. Int J Environ Res Public Health 2017;14:369.

21. Song Y, Wang HJ, Dong B, et al. 25-year trends in gender disparity for obesity and overweight by using WHO and IOTF definitions among Chinese school-aged children: a multiple cross-sectional study. BMJ Open 2016;6:e011904.

22. Zhao P, Gu X, Qian D, et al. Socioeconomic disparities in abdominal obesity over the life course in China. Int J Equity Health 2018;17:96.

23. Wang Y, Mi J, Shan XY, et al. Is China facing an obesity epidemic and the consequences? The trends in obesity and chronic disease in China. Int J Obes (Lond) 2007;31:177-88.

24. Rothman KJ. BMI-related errors in the measurement of obesity. Int J Obes (Lond) 2008;32 Suppl 3:S56-9.

25. Gómez-Ambrosi J, Silva C, Galofre JC, et al. Body mass index classification misses subjects with increased cardiometabolic risk factors related to elevated adiposity. Int J Obes (Lond) 2012;36:286-94.

26. Must A, Anderson SE. Body mass index in children and adolescents: considerations for population-based applications. Int J Obes (Lond) 2006;30:590-4.

27. Ogden CL, Fryar CD, Hales CM, et al. Differences in Obesity Prevalence by Demographics and Urbanization in US Children and Adolescents, 2013-2016. JAMA 2018;319:2410-8.

Cite this article as: Zhao S, Li X, Xiang ST, Xie L, Kang R, Li L, Xiao Z, Zhong Y. Changes in the age-specific body mass index distribution among urban children between 2002 and 2018 in Changsha, China. Transl Pediatr 2021;10(3):502-509. doi: $10.21037 /$ tp-20-305 


\section{Supplementary}
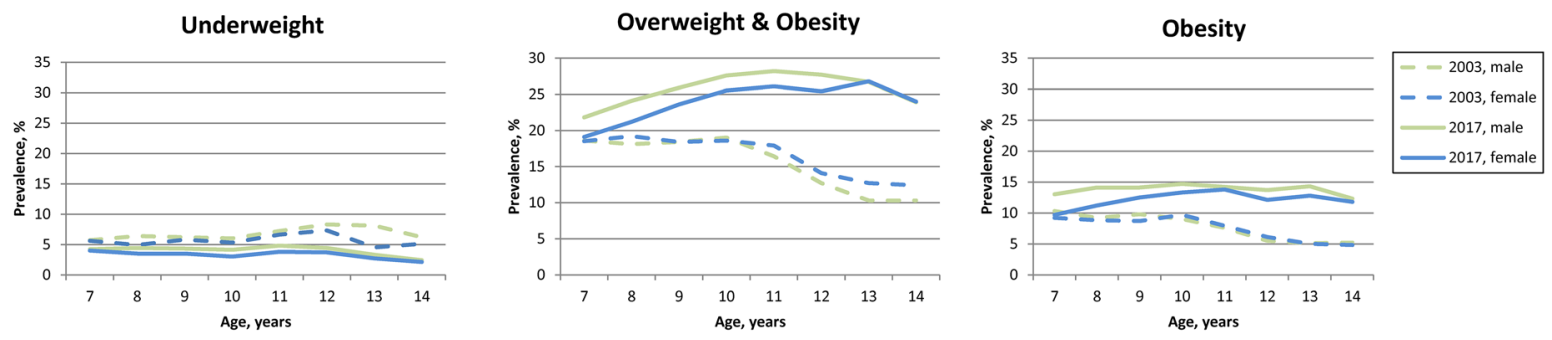

Figure S1 Age and sex-specific prevalence of underweight, overweight, and obesity among children in 2003 and 2017, Yuhua District, Changsha, China.
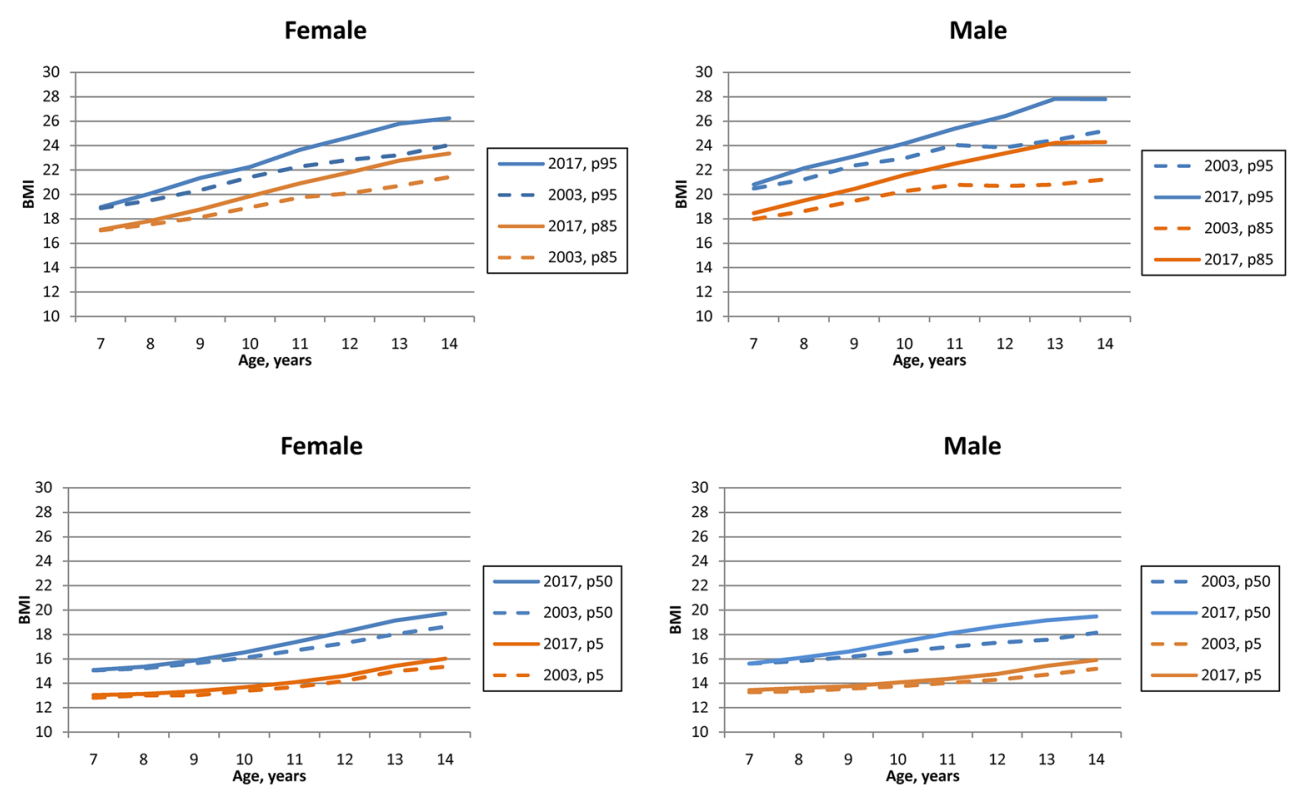

Figure S2 Distribution of BMI at 5th, 50th, 85th, and 95th percentile by age and sex among children from Yuhua District, Changsha, China in 2003 and 2017. p5, 5th percentile; p50, 50th percentile; p85, 85th percentile; p95, 95th percentile. BMI, body mass index. 
Table S1 Standardized rates of underweight, healthy weight, overweight, and obesity in 2003 and 2017 among children 7-14 years, Yuhua District, Changsha, China

\begin{tabular}{|c|c|c|}
\hline Weight status and sex & \multicolumn{2}{|c|}{ Standardized rates ${ }^{\mathrm{a}}, \%(95 \% \mathrm{Cl})$} \\
\hline \multicolumn{3}{|l|}{ Underweight } \\
\hline Both sexes & $6.2(6.0,6.4)$ & $3.8(3.7,3.9)$ \\
\hline Male & $6.6(6.4,6.9)$ & $4.1(3.9,4.3)$ \\
\hline \multicolumn{3}{|l|}{ Healthy weight } \\
\hline Both sexes & $77.1(76.7,77.4)$ & $71.5(71.1,71.8)$ \\
\hline Male & $77.0(76.5,77.5)$ & $70.2(69.7,70.6)$ \\
\hline Female & $77.2(76.7,77.7)$ & $73.0(72.5,73.4)$ \\
\hline Male & $8.2(7.9,8.5)$ & $11.8(11.5,12.1)$ \\
\hline Female & $9.2(8.8,9.5)$ & $11.6(11.2,11.9)$ \\
\hline \multicolumn{3}{|l|}{ Obesity } \\
\hline Both sexes & $8.1(7.9,8.3)$ & $13.1(12.8,13.3)$ \\
\hline Male & $8.2(7.9,8.5)$ & $13.9(13.6,14.2)$ \\
\hline Female & $8.0(7.7,8.3)$ & $12.1(11.7,12.4)$ \\
\hline
\end{tabular}

${ }^{a}$, rates were adjusted by age and sex, using the sum of both population as the standard population. $\mathrm{Cl}$, confidence interval. 